\title{
Starving the synovium: angiogenesis and inflammation in rheumatoid arthritis
}

\author{
Gary S. Firestein \\ Division of Rheumatology, Allergy and Immunology, University of California, San Diego School of Medicine, La Jolla, \\ California 92093-0656, USA \\ Address correspondence to: Gary S. Firestein, Division of Rheumatology, Allergy and Immunology, \#0656, University of California, \\ San Diego School of Medicine, 9500 Gilman Drive, La Jolla, California 92093-0656, USA. Phone: (619) 822-0480; Fax: (619) 534-2606; \\ E-mail: gfirestein@ucsd.edu
}

Traditional paradigms for rheumatoid arthritis (RA) have implicated a variety of mechanisms that contribute to the initiation and perpetuation of synovial inflammation, including T-cell activation, persistence of cytokine networks, and production of proinflammatory small molecules (1). Understanding these pathways has led to novel, and in some cases, strikingly effective treatments. For instance, clinical and basic science research demonstrating the importance of tumor necrosis factor- $\alpha$ (TNF- $\alpha$ ) in synovial inflammation led to the successful use of tumor necrosis factor (TNF)- $\alpha$ inhibitors in RA (2).

Alternative perspectives on synovial biology have recently suggested new approaches that borrow freely from other disciplines, especially oncology. Rather than treating the rheumatoid synovium as an inert infrastructure through which inflammatory cells aimlessly wander, it can also be viewed as a multicentric tumor-like mass that invades and destroys its local environs. For instance, the synovium contains specialized cells called synoviocytes that exhibit characteristics of tumor cells in $\mathrm{RA}$, including somatic mutations in key regulatory genes like $\mathrm{H}$-ras and the p53 tumor suppressor (3-6). This notion, originally suggested by Fassbender many years ago (reviewed in ref. 7), implies that one might address the expanding inflammatory synovium by targeting its source of nutrients in much the same way one might approach a locally invasive tumor.

There are several potential mechanisms whereby suppression of blood vessel growth could provide benefit in arthritis (Table 1). First, diminishing blood supply interferes with the care and feeding of a growing tissue burdened by substantial metabolic requirements. This rationale has typically been associated with neoplasms. Although its applicability to RA is unproven, the ability to starve the synovium has considerable appeal in light of its partially transformed characteristics. Second, decreasing the vasculature in an inflamed tissue minimizes the route of ingress for immune cells into the synovium. Blood vessels, especially high endothelial vessels, in rheumatoid synovium express a panoply of adhesion molecules that summon inflammatory cells into the joint. Very late activation antigen (VLA)-4 counterreceptors (like vascular cell adhesion molecule[VCAM]-1 and CS1 fibro-nectin), $\beta 2$ integrin ligands (like intercellular adhesion molecule [ICAM]), and E- and Pselectin are abundant in inflamed synovium and contribute to lymphocyte, monocyte, and neutrophil recruitment (8). Finally, elimination of endothelial cells deletes a potent source of proinflammatory cytokines, chemo-kines, and small molecules that play a pivotal role in synovitis. Chemoattractants like platelet activating factor and interleukin-8 (IL-8) are important mediators produced by rheumatoid microvascular endothelium that contribute to leukocyte adhesion and migration.

Blood vessel growth and involution has been studied extensively in RA. On first blush, there appears to be a remarkable increase in vascular density in the synovium. Despite this rich blood supply, the rheumatoid synovium remains a rather inhospitable environment, with marked hypoxia and aci- dosis. Careful capillary morphometry suggests that the growth of the synovial mass actually outstrips neovascularization, thereby exacerbating local ischemia (9). Also, repetitive extension and flexion can contribute to ischemiareperfusion injury in an inflamed joint. Accumulation of activated macro-phages and neutrophils provides an additional source of noxious reactive oxygen and nitrogen species in the joint. This, along with increased metabolic demands of a highly catabolic tissue and decreased supply resulting from elevated intra-articular pressure and capillary collapse, leads to the local generation of angiogenesis factors that support further blood vessel growth (10). For instance, vascular endothelial growth factor (VEGF) is highly expressed in the synovial intimal lining and is produced by fibroblastlike synoviocytes that have been exposed to hypoxia and IL-1. Additional angiogenesis factors, like fibroblast growth factor (FGF), TNF- $\alpha$, and soluble E-selectin, are also produced in the rheumatoid joint and contribute to vascular proliferation.

Blood vessel growth, therefore, is a dynamic process in the inflamed joint and appears to result from local hypoxia and growth factor production. Proliferation markers are expressed by many dividing endothelial cells in rheumatoid synovium under the influence of these mediators (11). The new blood vessels in RA synovium express $\alpha v / \beta 3$ integrin,
Table 1

Potential mechanisms of anti-angiogenesis treatment in arthritis

1. Diminish nutrient supply to tumor-like synovium.

2. Inhibit leukocyte adhesion and migration by decreasing endothelial cell surface area.

3. Decrease chemokine and cytokine production by activated endothelial cells. 
which is essential to blood vessel formation in wounds and tumors (12). Notably, $\alpha \mathrm{v} / \beta 3$ blockade interferes with angiogenesis in neoplastic diseases and suppresses tumor growth. DNA damage (likely due to locally produced reactive oxygen and nitrogen) and apoptosis are also evident in rheumatoid synovial endothelium; this suggests that the vasculature is constantly remodeling ( 9 , 13). Anti-angiogenesis factors like thrombospondin are present in the joint but do not co-localize with the involuting vessels.

The benefit of agents that suppress neovascularization in arthritis was first demonstrated by Brahn and colleagues (14) when they reported remarkable efficacy of the fumagillin derivative AGM-1470 (TNP-470). This compound, which is toxic to proliferating endothelial cells, prevented arthritis and reversed established disease in both adjuvant arthritis and collagen-induced arthritis in rats. In addition to decreasing inflammation, bone and cartilage damage were also suppressed. Taxol, which can induce endothelial cell apoptosis, was also effective in an animal model of arthritis (15). Vascular corrosion casts of arthritic rat synovium revealed dramatic expansion of the blood vessel volume, an extensive interconnecting architecture, and vascular arrays like Gaudi cathedrals spiraling toward the synovial lining. In Taxoltreated animals, the neovascular components reverted to the normal synovial morphology as arthritis diminished.

An article in this issue of the JCI describes an alternative approach to angiogenesis blockade in arthritis using an $\alpha v / \beta 3$ integrin inhibitor in a rabbit model of arthritis (16). As noted above, this integrin is intimately involved in blood vessel growth, especially in wound healing and neoplasms. Similar to RA synovium, $\alpha \mathrm{v} / \beta 3$ is expressed by proliferating blood vessels in inflamed rabbit synovium. Using a cyclic arginine-glycine-aspartic acid (RGD) peptide, modest clinical efficacy was demonstrated in both preventative and therapeutic dosing regimens. Decreased synovial inflammation and increased endothelial cell apoptosis was evident in the treated animals.

Most intriguing, bone and cartilage protection was more impressive than the effect on standard measures of inflammation like joint swelling. Because tumor-like invasive pannus is responsible for much of the joint damage, the data suggest that the analogy to locally expansive neoplasia might not be farfetched. A poor correlation between synovial inflammation and joint destruction has also been observed in RA, and effective anti-inflammatory agents often have little or no influence on disease progression. The mechanism of action for the $\alpha \mathrm{v} / \beta 3$ antagonist is still a matter of some debate, and there are no data to distinguish which of the possibilities outlined in Table 1 is the most important in arthritis.

What does this teach us about blood vessels as a target for future therapeutic intervention? At the very least, it provides additional solid rationale for pursuing anti-angiogenesis avenues in patients with RA. The potential for toxicity is likely modest, because the need for neovascularization is minimal in adults (aside from wound healing), and alternative pathways to blood vessel growth could exist. Although deletion of the $\alpha \mathrm{v}$ gene in mice is usually lethal in utero, extensive vascular growth and organogenesis still occur (17). This suggests that non- $\alpha_{\mathrm{V}}$ receptor-ligand pairs might also regulate angiogenesis, at least during embryonic development. Effective anti-angiogenesis treatment in arthritis also provides insight into the delicate balance between blood supply and demand in an inflamed, locally invasive tissue. There is already some evidence that interfering with blood vessel proliferation might have beneficial effects in human RA. Anti-TNF- $\alpha$ antibody administration leads to marked clinical improvement, and synovial biopsies from treated patients show a significant decrease in vascularity (18). It is not certain if this is due to a direct effect on TNF- $\alpha$, which has angiogenic activity, or a secondary action on the production of other angiogenesis factors like VEGF and FGF. In either case, the prospect of blood vessel-directed therapy has enormous clinical potential in synovial inflammatory disease.

1. Firestein, G.S. 1996. Etiology and pathogenesis of rheumatoid arthritis. In Textbook of rheumatology. 5 th ed. W.N. Kelley, E.D. Harris, Jr., S. Ruddy, and C.B. Sledge, editors. W.B. Saunders. Philadelphia, PA. 851-897.

2. Moreland, L.W., et al. 1997. Treatment of rheumatoid arthritis with a recombinant human tumor necrosis factor receptor (p75)-Fc fusion protein. N. Engl. J. Med. 337:141-147

3. Firestein, G.S. 1996. Invasive fibroblast-like synoviocytes in rheumatoid arthritis: passive responders or transformed aggressors? Arthritis Rheum. 39:1781-1790.

4. Roivainen, A., et al. 1998. H-ras oncogene point mutations in arthritic synovium. Arthritis Rheum. 40:1636-1643.

5. Firestein, G.S., Echeverri, F., Yeo, M., Zvaifler, N.J. and Green, D.R. 1997. Somatic mutations in the p53 tumor suppressor gene in rheumatoid arthritis synovium. Proc. Natl. Acad. Sci. USA. 94:10895-10900.

6. Reme, T., et al. 1998. Mutations of the p53 tumour suppressor gene in erosive rheumatoid synovial tissue. Clin. Exp. Immunol. 111:353-358.

7. Fassbender, H.G., and Gay, S. 1988. Synovial processes in rheumatoid arthritis. Scand. J. Rheumatol. 76(Suppl.):1-7.

8. Cronstein, B.N., and Weissmann, G. 1993. The adhesion molecules of inflammation. Arthritis Rheum. 36:147-157.

9. Stevens, C.R., Blake, D.R., Merry, P., Revell, P.A., and Levick, J.R. 1991. A comparative study by morphometry of the microvasculature in normal and rheumatoid synovium. Arthritis Rheum. 34:1508-1513.

10. Koch, A.E. 1998. Angiogenesis: implications for rheumatoid arthritis [review]. Arthritis Rheum. 41:951-962.

11. Walsh, D.A., Wade, M., Mapp, P.I., and Blake, D.R. 1998. Focally regulated endothelial proliferation and cell death in human synovium. Am. J. Pathol. 152:691-702.

12. Brooks, P.C., Clark, R.A., and Cheresh, D.A. 1994 Requirement of vascular integrin alpha v beta 3 for angiogenesis. Science. 264:569-571.

13. Firestein, G.S., Yeo, M., and Zvaifler, N.J. 1995 Apoptosis in rheumatoid arthritis synovium. J. Clin. Invest. 96:1631-1638.

14. Peacock, D.J., Banquerigo, M.L., and Brahn, E. 1992. Angiogenesis inhibition suppresses collagen arthritis. J. Exp. Med. 175:1135-1138.

15. Arsenault, A.L., Lhotak, S., Hunter, W.L., Banquerigo, M.L., and Brahn, E. 1998. Taxol involution of collagen-induced arthritis: ultrastructural correlation with the inhibition of synovitis and neovascularization. Clin. Immunol. Immunopathol. 86:280-289.

16. Storgard, C.M., et al. 1999. Decreased angiogenesis and arthritic disease in rabbits treated with an $\alpha \mathrm{v} / \beta 3$ antagonist. J. Clin. Invest. 103:47-54

17. Bader, B.L., Rayburn, H., Crowley, D., and Hynes, R.O. 1998. Extensive vasculogenesis, angiogenesis, and organogenesis precede lethality in mice lacking all $\alpha_{\mathrm{V}}$ integrins. Cell. 95:507-519.

18. Taylor, P.C., et al. 1998. Reduced synovial vascularity following TNF- $\alpha$ blockade in rheumatoid arthritis. Arthritis Rheum. 41(Suppl.):S295. 